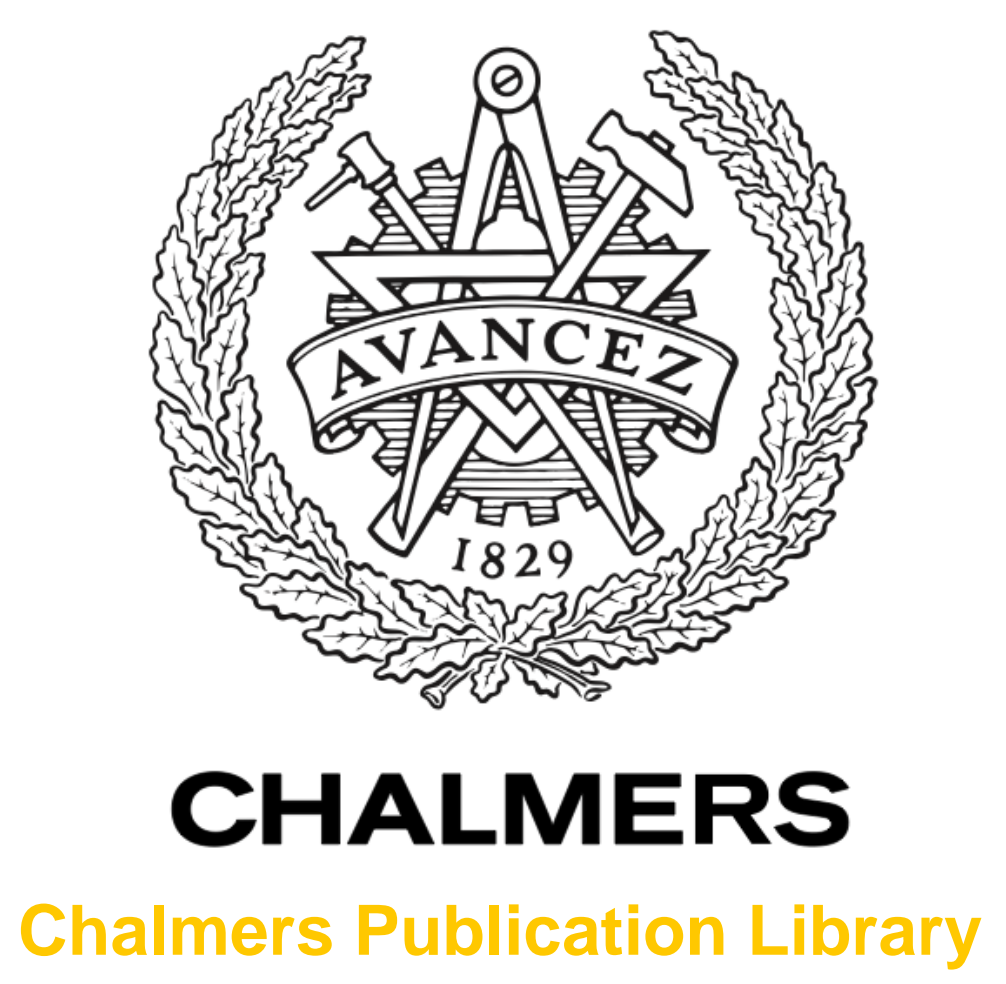

\title{
Automotive Threat Assessment Design for Combined Braking and Steering Maneuvers
}

This document has been downloaded from Chalmers Publication Library (CPL). It is the author's version of a work that was accepted for publication in:

IEEE Transactions on Vehicular Technology (ISSN: 0018-9545)

Citation for the published paper:

Ali, M. ; Gelso, E. ; Sjöberg, J. (2013) "Automotive Threat Assessment Design for Combined Braking and Steering Maneuvers". IEEE Transactions on Vehicular Technology, vol. PP(99), pp. 1.

http://dx.doi.org/10.1109/TVT.2012.2231444

Downloaded from: http://publications.lib.chalmers.se/publication/174540

Notice: Changes introduced as a result of publishing processes such as copy-editing and formatting may not be reflected in this document. For a definitive version of this work, please refer to the published source. Please note that access to the published version might require a subscription.

Chalmers Publication Library (CPL) offers the possibility of retrieving research publications produced at Chalmers University of Technology. It covers all types of publications: articles, dissertations, licentiate theses, masters theses, conference papers, reports etc. Since 2006 it is the official tool for Chalmers official publication statistics. To ensure that Chalmers research results are disseminated as widely as possible, an Open Access Policy has been adopted.

The CPL service is administrated and maintained by Chalmers Library. 


\title{
Automotive Threat Assessment Design for Combined Braking and Steering Maneuvers
}

\author{
Mohammad Ali ${ }^{\mathrm{ab}}$, Esteban Gelso ${ }^{\mathrm{b}}$ and Jonas Sjöberg ${ }^{\mathrm{b}}$
}

\begin{abstract}
The active safety systems available on the passenger cars market today, automatically deploy automated safety interventions in situations where the driver is in need of assistance. In this paper, we consider the process of determining whether such interventions are needed. In particular, we design a threat assessment method which evaluates the risk that the vehicle will either leave the road or its maneuverability will be significantly reduced within a finite time horizon. The proposed threat assessment method accounts for combined braking and steering maneuvers, which results in a nonlinear dynamical vehicle behavior. We formulate the threat assessment problem as a nonconvex constraint satisfaction problem and implement an algorithm that solves it through interval-based consistency techniques. Experimental validation of the proposed approach indicates that constraint violation can be predicted, while avoiding the detection of false threats.
\end{abstract}

\section{INTRODUCTION}

This paper considers the threat assessment problem in automotive driver assistance systems. In particular, we consider automotive safety systems with the capability of activating automated safety interventions in case there is a risk that the vehicle will depart the road. According to [1], roadway departure accidents account for approximately half of traffic related fatalities [1]. Several systems that attempt reducing such accident either through warnings or interventions have therefore been proposed [2, 3]. A common problem in safety systems is the problem of determining whether a situation is critical such that an automated intervention needs to be activated. We refer to this problem as the threat assessment problem.

A challenging aspect of the threat assessment problem is that it is inherently associated with potentially conflicting objectives. On one hand, safety systems need to detect critical situations and adequately assist the driver whenever this is necessary in order to ensure vehicle safety. On the other hand, alerts or interventions which drivers consider unnecessary, contribute negatively to their confidence in such systems. A highlighting example of this, is the case where a safety system suddenly performs an unmotivated full braking intervention. If such an unmotivated intervention occurs, the driver's confidence in the safety system would be seriously compromised. In commercially available safety systems, interventions are therefore often suppressed in uncertain situations and issued only once accidents have become unavoidable for the driver.

Copyright (c) 2012 IEEE. Personal use of this material is permitted. However, permission to use this material for any other purposes must be obtained from the IEEE by sending a request to pubs-permissions@ieee.org. ${ }^{a}$ Active Safety and Chassis, Volvo Car Corporation. ${ }^{b}$ Department of Signals and Systems, Chalmers University of Technology.
In [4], we presented a model based threat assessment method, specifically accounting for limitations in the vehicle's and the driver's ability in safely driving the vehicle. We proposed a solution to the problem of evaluating whether an admissible steering maneuver exists, that can drive the vehicle, while keeping it within a prescribed subset of the state and input space, where the driver is deemed capable of preserving vehicle safety. The underlying idea is that, if such a steering maneuver does not exist, the driver can be deemed incapable of maintaining safety without assistance and an autonomous assisting intervention is thus motivated.

In this paper we extend the problem formulation and instead propose a solution to the problem of evaluating whether an admissible combined steering and braking maneuver exists, that can drive the vehicle while maintaining it within a prescribed subset of the state and input space. Just like in [4], the underlying idea is that, if such a maneuver does not exist, the driver can be deemed incapable of maintaining safety without assistance. By excluding the possible existence of combined maneuvers, the risk for unwanted interventions is even further reduced and autonomous assisting interventions are thus even more motivated.

Although the reachability analysis tools used to develop the method we proposed in [4] are powerful, they are restricted to linear (and piece-wise affine) systems with polyhedral constraints. Dynamical models that simultaneously capture a vehicle's longitudinal and lateral dynamics are however, in general, nonlinear. In the design of threat assessment algorithms that account for combined braking and steering, the restriction to reachability analysis tools for linear systems can thus be limiting. For systems with nonlinear dynamics and possibly nonlinear, non-convex constraints, reachable sets are more difficult to compute. In [5], the reachable set for a nonlinear system is approximated by considering a large number of candidate trajectories generated using rapidly-exploring random trees. This method can generate a large number of candidate trajectories but the resulting reachable set is always a subset of the true reachable set. A different approach is considered in [6], where an algorithm for computing the backward reachable set for a nonlinear system is presented. The approach proposed in [6] however requires the solution of a time-dependant partial differential equation which, like many other approaches, is associated with high memory and computational costs. A discussion on algorithms for computing reachable sets for complex systems is provided in [7].

In the approach presented here, we reformulate our threat assessment problem as a constraint satisfaction problem with nonlinear equality constraints. This is a non-convex problem 
formulation. In solving this problem we resort to intervalbased consistency techniques, which have been applied in several different domains. Examples are model-based fault detection, model-based fault diagnosis, robust control and robotics, see e.g. [8].

When using interval techniques the solution sets (see Definition 11) are represented by one or several intervals or boxes. By restricting the sets to this limited structure, the interval based methods can be used to obtain approximative solutions to nonconvex constraint satisfaction problems, enabling the possibility to utilize nonlinear models and constraints. As will be shown in Section IV, these solutions can be arbitrarily close to the true solutions if sufficient computational resources are available. Nevertheless, in reality computational resources are always limited and the interval based approaches offer a flexible trade-off between computation time and accuracy. Iteratively they improve the accuracy of the approximate solution and this can continue until the computational time is finished. Then a solution, which is guaranteed to enclose the true solution is returned. This means that a result is returned even when the available computational time is insufficient to achieve the desired accuracy. For algorithms that run in realtime, this is an important benefit.

The rest of the paper is organized as follows. Section $\amalg$ presents the models used in the threat assessment algorithm to describe the vehicle behavior. In Section III, the threat assessment problem is formulated as a constraint satisfaction problem. Section IV introduces the fundamental concepts of interval analysis. The proposed algorithm for the threat assessment problem is presented in Section V. In Section VI we present experimental results obtained with the proposed algorithm. Finally, in Section VII we close the paper with final remarks.

\section{MATHEMATICAL MODELS}

To describe the vehicle motion within the lane, we use a standard single-track vehicle model, illustrated in Figure 1 Consider the following differential equations,

$$
\begin{aligned}
m \dot{v}_{x} & =m v_{y} \dot{\psi}+2\left[F_{x_{f}}+F_{x_{r}}\right], \\
m \dot{v}_{y} & =-m v_{x} \dot{\psi}+2\left[F_{y_{f}}+F_{y_{r}}\right], \\
J_{z} \ddot{\psi} & =2\left[l_{f} F_{y_{f}}-l_{r} F_{y_{r}}\right], \\
\dot{e}_{\psi} & =\dot{\psi}-\dot{\psi}_{d}, \\
\dot{e}_{y} & =v_{y} \cos \left(e_{\psi}\right)+v_{x} \sin \left(e_{\psi}\right),
\end{aligned}
$$

where, $m$ and $J_{z}$ denote the vehicle mass and yaw inertia, respectively, $F_{y_{f}}, F_{y_{r}}$ are the lateral tire forces at the front and rear axles, respectively, $F_{x_{f}}, F_{x_{r}}$ are the longitudinal tire forces at the front and rear axles, respectively and $v_{x}$ and $v_{y}$ denote the vehicle's longitudinal and lateral velocity components, respectively. $\psi$ denotes the vehicle direction of travel in a fixed global frame and $\dot{\psi}$ denotes the vehicle rotation rate around a vertical axis located at the vehicle's center of gravity. $l_{f}$ and $l_{r}$ denote the distances of the front and rear axles, respectively, from the vehicle center of gravity as shown in Figure 1. $e_{y}$ denotes the distance of the vehicle center of gravity from the road centerline. $\psi_{d}$ is the orientation

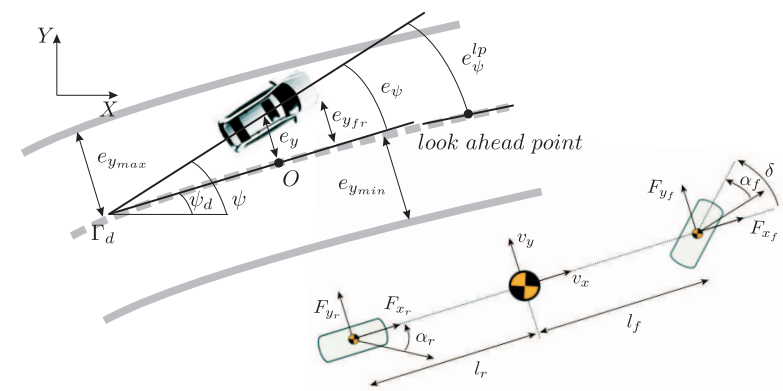

Fig. 1. Vehicle modeling notation.

of the road centerline, i.e., the orientation of the tangent to the curve $\Gamma_{d}$ in the point $O$ in Figure 1 and $e_{\psi}=\psi-\psi_{d}$ is the vehicle orientation in the lane.

In a real-time application, the road curvature $c(s)$, where $s$ denotes distance along the road ahead of the vehicle might be obtained from a digital map or through a vision system. The sensing technologies in e.g. [9] can be used for this purpose. Further, assuming $\dot{s} \approx v_{x}$, the exogenous disturbance signal $\dot{\psi}_{d}$, can then be approximated through the relation $\dot{\psi}_{d}=c v_{x}$. Hence, we make the following assumption.

Assumption 1: We assume an estimate of $\dot{\psi}_{d}$ is available over a future finite time horizon.

Forces acting on the vehicle are generated at the contact patch between tire and road. We denote by $f_{x_{i}}$ and $f_{y_{i}}$ the force components acting along the longitudinal and lateral tire axis, which lead to the following longitudinal and lateral force components in the vehicle body frame,

$$
\begin{aligned}
& F_{x_{f}}=f_{x_{f}} \cos (\delta)-f_{y_{f}} \sin (\delta), F_{x_{r}}=f_{x_{r}}, \\
& F_{y_{f}}=f_{x_{f}} \sin (\delta)+f_{y_{f}} \cos (\delta), F_{y_{r}}=f_{y_{r}} .
\end{aligned}
$$

We assume the vehicle is front wheel driven and calculate the longitudinal force components as,

$$
\begin{array}{lll}
f_{x_{f}}=\rho f_{x}, & f_{x_{r}}=(1-\rho) f_{x}, & \text { if } f_{x} \leq 0 \\
f_{x_{f}}=f_{x}, & f_{x_{r}}=0, & \text { if } f_{x} \geq 0
\end{array}
$$

where $\rho$ gives the brake distribution between the front and rear axle imposed by the design of the brake system and the total longitudinal force $f_{x}$ is considered as an input signal.

The lateral tire force components are computed using a simplified version of the magic tire formula [10],

$$
\begin{aligned}
& f_{y_{i}}=\varphi_{i} f_{y 0_{i}}, f_{y 0_{i}}=\mu F_{z_{i}} \sin \left(C_{i} \arctan \left(B_{i} \alpha_{i}\right)\right), \\
& \varphi_{i}=\frac{\sqrt{\left(\mu F_{z_{i}}\right)^{2}-f_{x_{i}}^{2}}}{\mu F_{z_{i}}}, \quad i \in\{f, r\},
\end{aligned}
$$

where $B_{i}$ and $C_{i}$ are stiffness and shape coefficients, respectively, at the two axles, $\alpha_{i}$ are tire slip angles, $\mu$ is the friction coefficient and $F_{z_{i}}$ denotes the normal force. $f_{y 0_{i}}$ is the lateral force in pure cornering conditions. However, simultaneous acceleration and cornering results in a reduced lateral force. This effect is modeled by multiplying $f_{y 0_{i}}$ with $\varphi_{i}$ in (4), [10].

Assuming small angles, the tire slip angles are approximated as,

$$
\alpha_{f}=\frac{v_{y}+l_{f} \dot{\psi}}{v_{x}}-\delta, \quad \alpha_{r}=\frac{v_{y}-l_{r} \dot{\psi}}{v_{x}},
$$


where $\delta$ denotes the steering angle at the front wheel and is also considered an input signal.

The friction coefficient $\mu$ in (4) is considered an exogenous disturbance signal.

Assumption 2: In this paper, at each time instant, we will assume an estimate of $\mu$ is available and this is kept constant over a finite time horizon.

In general, friction estimation is however difficult and requires high excitation of the vehicle dynamics. In addition, the most common estimation techniques, based on vehicle dynamics signals, provide estimates of the friction coefficient at the current position but not for the coming road. See e.g. [11, 12] for an overview on friction estimation techniques.

We write the model (1)-(5) in the following compact form,

$$
\dot{\mathbf{x}}(t)=f(\mathbf{x}(t), \mathbf{u}(t), \mathbf{w}(t)),
$$

where $\mathbf{x}=\left[v_{x}, v_{y}, \dot{\psi}, e_{\psi}, e_{y}\right]^{T}, \mathbf{u}=\left[f_{x}, \delta\right]^{T}$ and $\mathbf{w}=\left[\dot{\psi}_{d}, \mu\right]$ are the state, input and disturbance vectors respectively.

\section{A. Constraints}

In this section, we express the requirements that the vehicle stays in the lane while operating in a stable operating region as constraints on the vehicle state, input and disturbance variables.

Let $e_{y_{i j}}, i \in\{f, r\}, j \in\{l, r\}$, be the distances of the four vehicle corners from the lane centerline $\left(e_{y_{f r}}\right.$ is shown in Figure 1). The requirement that the vehicle stays in the lane is then expressed,

$$
-e_{y_{\max }} \leq e_{y_{i j}} \leq e_{y_{\max }} .
$$

In addition to staying in the lane, we require that the vehicle operates in a region of the state space where the vehicle is easily maneuverable by a normally skilled driver. The requirement that the vehicle operates in stable operating conditions is ensured by limiting the tire slip angles $\alpha_{i}$,

$$
\alpha_{i_{\min }} \leq \alpha_{i} \leq \alpha_{i_{\max }}, i \in\{f, r\} .
$$

In this region the vehicle behavior is predictable by most drivers and Electronic Stability Control (ESC) systems are inactive.

The driver can influence the vehicle's motion through the input signals $\delta$ and $f_{x}$. The force component $f_{x}$ is limited by the available friction and the steering wheel angle $\delta$ is subject to mechanical constraints imposed by the vehicle design. We will also assume that, for convenience purposes, under normal circumstances, the driver will not impose larger deceleration and steering rate than $a_{\max }, \dot{\delta}_{\max }$. We express these limitations as,

$$
\begin{gathered}
-\delta_{\max } \leq \delta \leq \delta_{\max }, \\
-\mu F_{z_{i}} \leq f_{x_{i}} \leq \mu F_{z_{i}}, \\
-m a_{\max } \leq f_{x} \leq 0 \\
-\dot{\delta}_{\max } \leq \dot{\delta} \leq \dot{\delta}_{\max }
\end{gathered}
$$

Rate limitations for the braking force are neglected.
The constraints (7)-9 can be compactly written as,

$$
h(\mathbf{x}(t), \mathbf{u}(t), \mathbf{w}(t)) \leq \mathbf{0}
$$

where $\mathbf{0}$ is a vector of zeros with appropriate dimension.

\section{THREAT ASSESSMENT AS A CONSTRAINT SATISFACTION PROBLEM}

In this section we formulate the threat assessment problem as a Constraint Satisfaction Problem (CSP). At each time instant, if the vehicle state does not satisfy the constraints 10 , the vehicle's operation can be considered unsafe. The threat assessment problem is therefore formulated as the problem of evaluating whether an admissible sequence of combined steering and braking maneuvers $\mathbf{u}$ exists, that can drive the system (6) over a future finite time horizon, while satisfying the constraints 10].

Denote by,

1) $\mathcal{V}=\left\{z_{1}, \ldots, z_{n}\right\}$, a set of numeric variables,

2) $\mathcal{D}=\left\{\mathcal{Z}_{1}, \ldots, \mathcal{Z}_{n}\right\}$, a set of domains where $\mathcal{Z}_{i}$, is the domain associated with the variable $z_{i}$,

3) $\mathcal{C}=\left\{C_{1}(\mathbf{z}), \ldots, C_{m}(\mathbf{z})\right\}$, a set of constraints where a constraint $C_{i}(\mathbf{z})$ is determined by a numeric relation (equation, inequality, inclusion, etc.) linking a set of variables under consideration.

We let $\mathcal{C S P}=(\mathcal{V}, \mathcal{D}, \mathcal{C})$, denote a CSP and introduce the following definition,

Definition 1: The solution of a CSP, $\operatorname{sol}(\mathcal{C S P})$ is the set of numerical variables $\Sigma$ for which all the constraints $C_{i} \in \mathcal{C}$ are satisfied, i.e.,

$$
\Sigma=\left\{\mathbf{z} \in \mathcal{Z} \mid C_{i}(\mathbf{z}) \text { holds } \forall C_{i} \in \mathcal{C}\right\} .
$$

The threat assessment CSP is formulated in discrete time, the continuous time system (6) is therefore discretized with a sampling time $T_{s}$ to obtain the discrete time constrained system,

$$
\begin{array}{r}
\mathbf{x}(k+1)=f^{d}(\mathbf{x}(k), \mathbf{u}(k), \mathbf{w}(k)), \\
h^{d}(\mathbf{x}(k), \mathbf{u}(k), \mathbf{w}(k)) \leq \mathbf{0} .
\end{array}
$$

The threat assessment CSP over a 1-step horizon can now be stated as,

$$
\begin{aligned}
\mathcal{V}=\{ & \mathbf{x}(k), \mathbf{x}(k+1), \mathbf{u}(k), \mathbf{u}(k+1), \mathbf{e}(k)\}, \\
\mathcal{D}=\{ & \left.\mathcal{X}_{k}, \mathcal{X}_{k+1}, \mathcal{U}_{k}, \mathcal{U}_{k+1}, \mathcal{E}_{k}\right\}, \\
\mathcal{C}=\{ & h^{d}(\mathbf{x}(k+1), \mathbf{u}(k+1), \mathbf{w}(k+1)) \leq \mathbf{0}, \\
& \mathbf{x}(k+1)=f^{d}(\mathbf{x}(k), \mathbf{u}(k), \mathbf{w}(k)), \\
& h^{d}(\mathbf{x}(k), \mathbf{u}(k), \mathbf{w}(k)) \leq \mathbf{0}, \\
& \tilde{\mathbf{x}}(k)=\mathbf{x}(k)+\mathbf{e}(k)\},
\end{aligned}
$$

where $\tilde{\mathbf{x}}(k)$ is the vector of state variables estimates and $\mathbf{e}(k)$ represents the uncertainty associated with the estimates. For each estimate, the uncertainty is considered unknown but bounded, i.e. $\mathbf{e}(k) \in \mathcal{E}_{k}$, for some bounded set $\mathcal{E}_{k}$. The symbols $\mathcal{X}_{k}, \mathcal{U}_{k}$, denote the domains associated with the state and input vectors at time step $k$, respectively. We note that, in the CSP (13), the disturbance signals $\mathbf{w}(k), \mathbf{w}(k+1)$ are not 
considered as part of the set of numerical variables $\mathcal{V}$. This is a consequence of the Assumptions 1 and 2 where estimates of the disturbance signal are assumed available. We remark that if the accuracy of the available sensor setup is poor, it is possible to account for uncertainties by including these signals in $\mathcal{V}$ and associated (uncertainty) domains $\mathcal{W}_{k}, \mathcal{W}_{k+1}$ in the set of domains $\mathcal{D}$. The $N$-step threat assessment CSP can be formulated by repetition of (13).

\section{SOLVING CONSTRAINT SATISFACTION PROBLEMS USING INTERVAL TECHNIQUES}

Several methods can be used to find the solution $\Sigma$ to a CSP (Definition 1). This section gives a brief introduction to interval based consistency techniques, which have been used in the results presented in this paper.

In interval-based consistency techniques, the solution to a CSP, $\Sigma$, is approximated by one or several intervals or boxes $[\mathbf{z}]_{i}$. The solution, $\Sigma$, is obtained by pruning the initial domain of the variables of the CSP, and through successive elimination of subboxes which cannot contain the solution. The consistency techniques most commonly used are known as Hull-consistency (also called 2B-consistency) and Boxconsistency, or are variations of them, [13]. In general, interval techniques are associated with wrapping of generic sets into boxes, decomposition of constraints and use of interval operations which leads to overestimation of the solution $\Sigma$. This overestimation can be done arbitrarily tight with the cost of increased computational time, as will be described in the following example. In this manuscript we utilize interval techniques which provide an outer approximation $\hat{\Sigma}$ that is guaranteed to enclose the true solution $\Sigma$. The choice of such techniques is commented in Section $\mathrm{V}$.

We illustrate the main functionality of the interval techniques through a simple example while for a rigorous treatment of consistency techniques for CSPs we refer the interested reader to [14, 8]. For the sake of clear and easy presentation, a simplified approach has been adopted in the example.

Example 1: Consider the nonlinear discrete time statespace model,

$$
\begin{aligned}
& x_{1}(k+1)=-0.32 \sqrt{x_{1}(k)}+x_{1}(k)+0.36 \sqrt{x_{2}(k)}, \\
& x_{2}(k+1)=-0.07 \sqrt{x_{2}(k)}+x_{2}(k),
\end{aligned}
$$

with its real variables $x_{1}$ and $x_{2}$ ranging in the domains $[0.3,0.36] \times[0.205,0.3]$, which defines $\mathcal{D}$. Assume that the state variables at $k=0$ belong to the intervals $\left[x_{1}(0)\right]=$ $[0.304,0.336]$ and $\left[x_{2}(0)\right]=[0.256,0.284]$, respectively. We are interested in computing the solution set $\Sigma$ at time instant $k=2$, i.e., $\{\mathbf{x}(k) \in \mathcal{D}$ with $k \in\{0,1,2\} \mid \mathbf{x}(k+1)=$ $\left.f^{d}(\mathbf{x}(k)), \mathbf{x}(0) \in[\mathbf{x}(0)]\right\}$, where $f^{d}(\mathbf{x}(k))$ is defined by (14).

We start from $[\mathbf{x}(0)]$ which in this example is already a box, hence no wrapping is needed. We utilize a natural inclusion function $\left[f^{d}\right]$ to propagate $[\mathbf{x}(0)]$ two time steps and obtain interval approximations of $\{\mathbf{x}(1), \mathbf{x}(2) \in \mathcal{D} \mid \mathbf{x}(k+1)=$ $\left.f^{d}(\mathbf{x}(k)), \mathbf{x}(0) \in[\mathbf{x}(0)]\right\}$. Figure $2(\mathrm{a})$ shows $[\mathbf{x}(0)]$, the computed approximations $[\mathbf{x}(1)]=\left[f^{d}\right]([\mathbf{x}(0)]),[\mathbf{x}(2)]=$ $\left[f^{d}\right]([\mathbf{x}(1)])$ and $\mathcal{D}$. We note that portions of $[\mathbf{x}(1)]$ and $[\mathbf{x}(2)]$

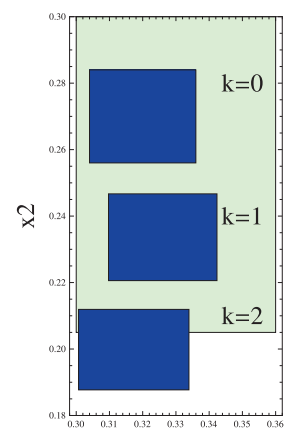

$\mathrm{x} 1$

(a)

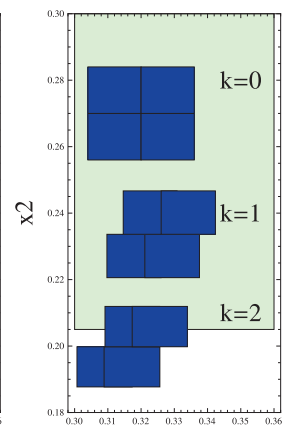

$\mathrm{x} 1$

(b)

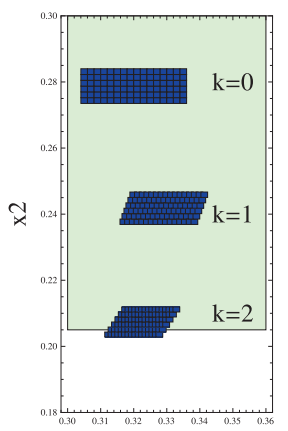

$\mathrm{x} 1$

(c)
Fig. 2. Approximated solution sets $\hat{\Sigma}$ for the problem considered in Example 1 with increasing accuracy from left to right. The outer approximation $\hat{\Sigma}$ of the solution $\Sigma$ is calculated using Box-consistency and a splitting step that generates sub-boxes by a succession of bisections of $\left[x_{1}(0)\right]$ and $\left[x_{2}(0)\right]$. The green (brighter) sets show the domain $\mathcal{D}$ and the blue (darker) sets show the solution sets at time steps $k=0,1,2$.

lie inside $\mathcal{D}$ hence they cannot be excluded from the solution set at this point. We also note that a portion of $[\mathbf{x}(2)]$ lies outside $\mathcal{D}$ which indicates that the initial region $[\mathbf{x}(0)]$ is potentially too large.

In order to improve the accuracy of the solution we split the initial box $[\mathbf{x}(0)]$ into four sub-boxes. We utilize the inclusion function $\left[f^{d}\right]$ again to propagate the four sub-boxes two time steps. The obtained results are shown in Figure 2(b). We note that, at $k=2$ two of the boxes are totally outside the domain $\mathcal{D}$. Consequently they can be excluded from the solution set $\hat{\Sigma}$ (for all $k$ ) and higher accuracy of the solution set can be obtained.

The process of splitting and propagating boxes can be continued until the desired accuracy has been reached, the solution is empty or the computational time is out. In Figure 2(c) the result after four divisions of the boxes is shown. Clearly, the solutions in Figure 2(c) are smaller and more accurate than the solutions in Figure 2(a)

\section{InterVAl-BASEd Threat ASSESSMEnt Algorithm}

In Section [II we formulated the threat assessment problem as a constraint satisfaction problem and in Section IV we showed how such problems can be solved with interval techniques. In this section we formulate the threat assessment algorithm which is to be repeatedly solved in an automotive safety system.

Denote by $\mathbf{W}_{k}=\left[\mathbf{w}_{k}, \mathbf{w}_{k+1}, \ldots, \mathbf{w}_{k+N-1}\right]$ a sequence of disturbance samples over the horizon $[k, k+N-1]$. We formulate an $N$-step threat assessment CSP and enforce the constraints (10) to hold for each time step over a finite time 


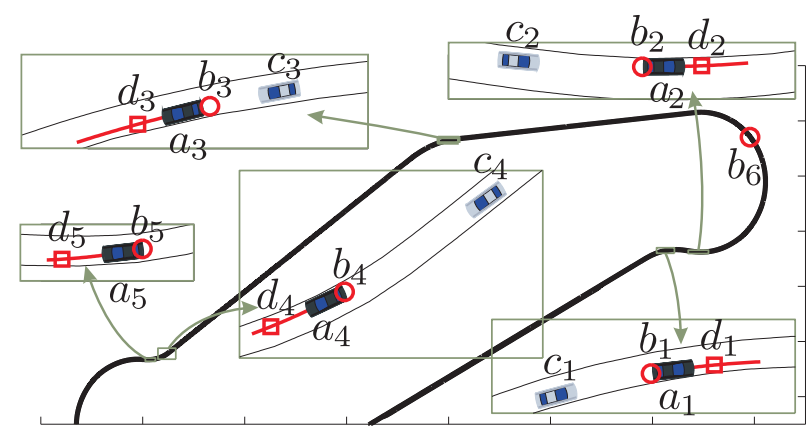

Fig. 3. Test track used to collect the experimental data. The darker color vehicle's denote vehicle positions at times $a_{i}$, the symbol o denotes the vehicle positions at times $b_{i}$, the brighter color vehicle's denote the vehicle positions at times $c_{i}$ and the symbol $\square$ denotes the vehicle positions at times $d_{i}$.

horizon of $N$ steps,

$$
\begin{aligned}
& \mathcal{V}=\{\mathbf{x}(k), \ldots, \mathbf{x}(k+N-1), \mathbf{u}(k), \ldots, \mathbf{u}(k+N-1), \\
& \quad \mathbf{e}(k)\} \\
& \mathcal{D}=\{[\mathbf{x}(k)], \ldots,[\mathbf{x}(k+N-1)],[\mathbf{u}(k)], \ldots, \\
& \quad[\mathbf{u}(k+N-1)],[\mathbf{e}(k)]\} \\
& \mathcal{C}=\left\{\mathbf{x}(i+1)=f^{d}(\mathbf{x}(i), \mathbf{u}(i), \mathbf{w}(i)), i=k, \ldots, k+N-2,\right. \\
& \quad h^{d}(\mathbf{x}(i), \mathbf{u}(i), \mathbf{w}(i)) \leq \mathbf{0}, i=k, \ldots, k+N-1, \\
& \quad \tilde{\mathbf{x}}(k)=\mathbf{x}(k)+\mathbf{e}(k)\} \\
& \mathcal{C} \mathcal{S P}_{T A}=(\mathcal{V}, \mathcal{D}, \mathcal{C})
\end{aligned}
$$

A threat assessment algorithm that sets a safety flag in case the threat assessment CSP (15) has an empty solution, hereby referred to as Algorithm 1, has been implemented. In Algorithm 1 an interval-based branch and prune algorithm is used to find the solution $\hat{\Sigma}=\operatorname{sol}\left(\mathcal{C S P}_{T A}\right)$, of the threat assessment CSP (15). As noted in Section IV, the solution $\hat{\Sigma}$ obtained with the interval solver is an outer approximation that encloses the true solution $\Sigma$, i.e., $\Sigma \subseteq \hat{\Sigma}$. Consequently $\hat{\Sigma}=\emptyset \Longrightarrow \Sigma=\emptyset$, hence, based on the model (12), a violation of the constraints (10) can be guaranteed within the horizon of $N$-steps if $\hat{\Sigma}$ is empty. In such case, a flag notSafe is set, activating an autonomous intervention or warning. Waiting until $\hat{\Sigma}=\emptyset$, reduces the risk of activating autonomous interventions in situations where the driver is not in need of assistance. However, if the difference between the sets $\hat{\Sigma}$ and $\Sigma$ is large, the autonomous intervention might be delayed which limits the effect of the intervention.

\section{EXPERIMENTAL RESULTS}

In order to validate the proposed threat assessment approach, experimental testing has been conducted at a test track located approximately $100 \mathrm{~km}$ outside Göteborg, Sweden. The test track is about $5 \mathrm{~km}$ long and is shown in Figure 3 Measurements of the state variables and disturbances were collected using a differential GPS unit, a built-in high precision inertial measurement unit along with a digital map. The test vehicle was driven several laps by a professional driver, which adopted both a normal and rougher driving style. In the normal driving case, the driver was asked to keep the posted speed limits, while in the rougher driving case, the driver was driving as fast as possible. During driving, the proposed threat assessment was inactive and the collected data has instead been post-processed through Algorithm 1 using a laptop PC. This enables the possibility to evaluate the performance of the threat assessment approach without influencing the vehicle motion and driver behavior through safety interventions. For the post-processing with Algorithm 1, no upper bound on the computational time was set.

The parameter values used are provided in Tables $\Pi$ and $\Pi$

TABLE I

VEHICLE MODEL PARAMETERS

\begin{tabular}{|l|l|l|l|}
\hline$m[\mathrm{~kg}]$ & $J_{z}\left[\mathrm{kgm}^{2}\right]$ & $l_{f}[\mathrm{~m}]$ & $l_{r}[\mathrm{~m}]$ \\
\hline 1695 & 2617 & 1.14 & 1.50 \\
\hline \hline$a[\mathrm{~m}]$ & $b[\mathrm{~m}]$ & $w[\mathrm{~m}]$ & $\mu[-]$ \\
\hline 1.83 & 2.69 & 1.77 & 1 \\
\hline \hline$B_{f}[-]$ & $B_{r}[-]$ & $C_{f}, C_{r}[-]$ & $\rho[-]$ \\
\hline-10.5 & -12.7 & 0.5 & 0.6 \\
\hline
\end{tabular}

TABLE II

DESIGN PARAMETERS

\begin{tabular}{|l|l|l|}
\hline$e_{y_{\max }}[\mathrm{m}]$ & $\alpha_{f_{\min }}, \alpha_{r_{\min }}\left[^{\circ}\right]$ & $\alpha_{f_{\max }}, \alpha_{r_{\max }}\left[{ }^{\circ}\right]$ \\
\hline 1.61 & -4 & 4 \\
\hline \hline$a_{\max }\left[\mathrm{m} / \mathrm{s}^{2}\right]$ & $\delta_{\max }\left[{ }^{\circ}\right]$ & $\dot{\delta}_{\max }\left[{ }^{\circ} / \mathrm{s}\right]$ \\
\hline 2 & 7 & 15 \\
\hline \hline$T s[\mathrm{~ms}]$ & $N[-]$ & \\
\hline 40 & 11 & \\
\hline
\end{tabular}

The parameters in Table Iare vehicle specific parameters while the parameters in Table $\amalg$ are design parameters. The desired behavior of a safety system is subjective and, just like with e.g. stability control systems, drivers have different preferences on activation timing and control authority. Large values of the bounds in Table [I delay interventions and can jeopardies safety while small values will lead to a system that intervenes often and might be perceived as intrusive. For a commercial application, it is possible to give drivers possibility to choose from a set of parameter configurations to accommodate the needs and preferences of different drivers. In this paper, the performance of the threat assessment algorithm has been tuned and evaluated based on its ability to predict constraint violations that actually occur and avoiding false constraint violation predictions, rather than relying on preferences of specific drivers. The bound $e_{y_{\max }}$ has been set by the road width and the rest of the design parameters in Table $\amalg$ have been tuned by balancing between maintaining a capability to detect threats while avoiding interventions when no constraint violation is imminent.

Uncertainties in the state estimates have been accounted for by setting,

$$
\begin{aligned}
& {[\mathbf{e}(k)]=\left[e_{1}(k)\right] \times\left[e_{2}(k)\right] \times\left[e_{3}(k)\right] \times\left[e_{4}(k)\right] \times\left[e_{5}(k)\right],} \\
& e_{i}(k)=\left[-0.05\left|\tilde{x}_{i}(k)\right|, 0.05\left|\tilde{x}_{i}(k)\right|\right], i \in\{1,2,3,4,5\} .
\end{aligned}
$$

where $\tilde{x}_{i}(k)$ denotes the $i$-th component of the measured state vector $\tilde{\mathbf{x}}(k)$. We remark that, potentially, the performance 


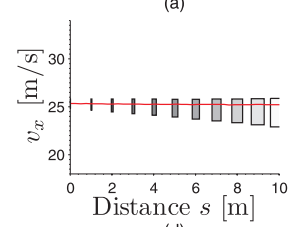

(d)
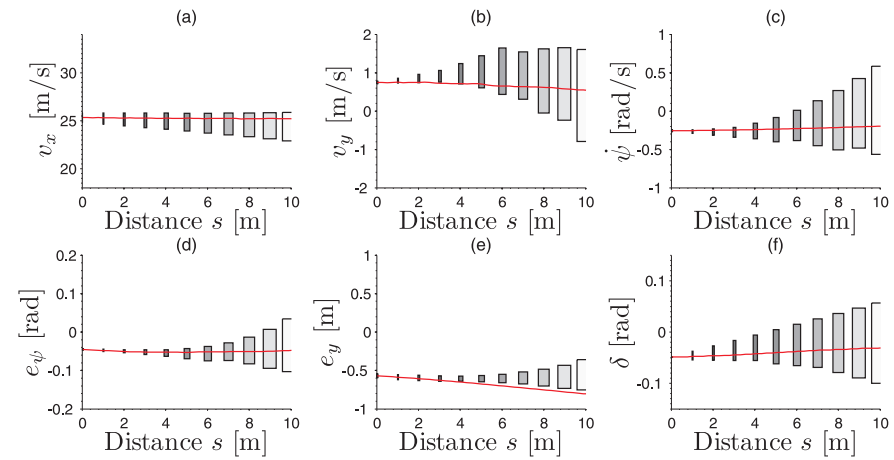

Fig. 4. (a)-(f) compare the approximated solution set $\hat{\Sigma}$ obtained through Algorithm 1 at position $b_{1}$ to measured vehicle states and steering angles. Each box shows predicted admissible variables for each step over the prediction horizon and the red solid line shows the actual trajectory traversed by the vehicle.

of the proposed threat assessment method could be further improved by utilizing knowledge about the measurement accuracy of the sensors used to acquire the state estimates.

The performance of the proposed algorithm where combined steering and braking maneuvers of the driver are considered is compared to a previously published algorithm where only steering is considered. We will refer to this alternative algorithm as Algorithm 2. In Algorithm 2, the vehicle model is linear and the threat assessment problem is then easier to solve. Details about the alternative algorithm are provided in [15]. The proposed threat assessment algorithm, Algorithm 1, proved capable of predicting violation of the constraints (10) within the prediction horizon without issuing any false detections in the considered dataset.

Next, we show results obtained in the situations illustrated in Figure 3, where the following notation is used: $a_{i}$ denotes a time instant where the Algorithm 1 returns notSafe $=1$, i.e., when the solution set $\hat{\Sigma}$ is empty. In Figure 3, the vehicle positions at times $a_{i}$ are marked out with a darker color vehicle. $b_{i}=a_{i}-100 \mathrm{~ms}$ and corresponding positions are marked with the symbol $\circ$ in Figure 3 These positions have been indicated for analysis purposes. $c_{i}$ are time instances where the alternative (steering only) threat assessment algorithm, Algorithm 2, predicts a constraint violation and the corresponding positions are marked with the brighter color vehicle in Figure 3 Finally, $d_{i}$ denotes a time instance where a violation of the constraints (10) occurs and corresponding positions are marked with the symbol $\square$.

Consider the time instances $d_{1}, d_{2}$ and $d_{3}$. At times, $d_{1}, d_{2}$, the vehicle is traveling at a speed of approximately $90 \mathrm{~km} / \mathrm{h}$ and at $d_{3}$ the speed is approximately $110 \mathrm{~km} / \mathrm{h}$. We note that, at these time instances, the vehicle violates the position constraints (7) by crossing the lane marking at the inner side of the curves. In Figure 3, the positions at the time instances $c_{1}, c_{2}, c_{3}$ indicate that Algorithm 2 predicts these situations somewhat early. At the times $c_{1}, c_{2}, c_{3}$ it is according to Algorithm 2 no longer possible to avoid a constraint violation by the adopted steering only approach. Figures 4,5 and 6 , show the solution sets $\hat{\Sigma}$ obtained by Algorithm 1, at times $b_{1}, b_{2}$ and $b_{3}$ respectively. At these time instances the (a)
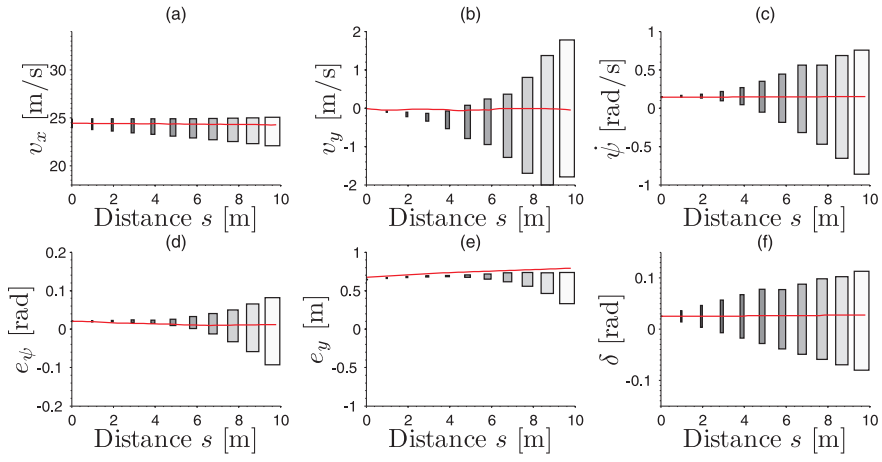

Fig. 5. (a)-(f) compare the approximated solution set $\hat{\Sigma}$ obtained through Algorithm 1 at position $b_{2}$ to measured vehicle states and steering angles. Each box shows predicted admissible variables for each step over the prediction horizon and the red solid line shows the actual trajectory traversed by the vehicle.
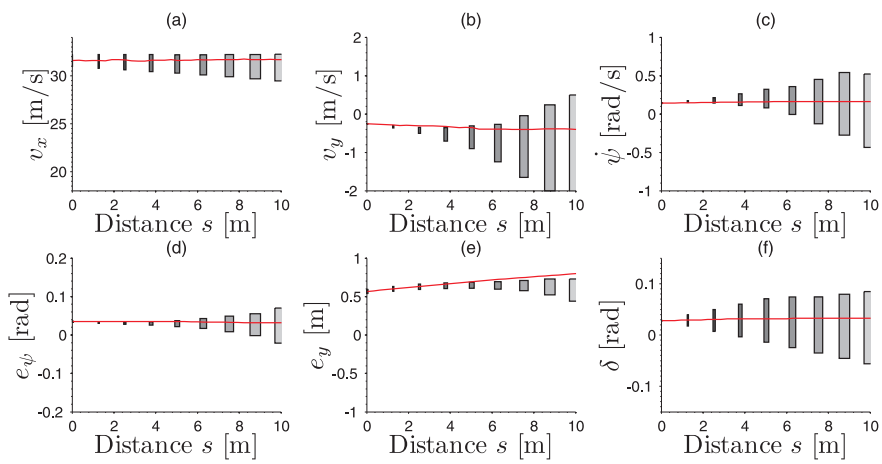

Fig. 6. (a)-(f) compare the approximated solution set $\hat{\Sigma}$ obtained through Algorithm 1 at position $b_{3}$ to measured vehicle states and steering angles. Each box shows predicted admissible variables for each step over the prediction horizon and the red solid line shows the actual trajectory traversed by the vehicle.

solution sets $\hat{\Sigma}$ are not empty which indicates that, potentially, there exists some combined braking and steering action that can keep the vehicle within the lane from these positions. Nevertheless, the Figures [4, 5] and 6 show that the driver chooses to maintain the high velocity and steering angle in these situations. Potentially, the driver was willing to risk slightly crossing the lane markings in order to be able to maintain a high speed throughout the curve, while keeping away from the outer lane border. Consequently at times $a_{1}, a_{2}$ and $a_{3}$ the solution sets $\hat{\Sigma}$ are empty, hence at these points, according to the assumed model and control limitations, the constraint violation has become unavoidable. At these time instances, it took the algorithm $690 \mathrm{~ms}, 300 \mathrm{~ms}$ and $<1 \mathrm{~ms}$, respectively, to conclude that $\hat{\Sigma}$ is empty.

If the available actuators can overcome the assumed control limitations of the driver, an assisting intervention might be issued in such situations in order to avoid the imminent constraint violation. In Figure 3 we note that an intervention based on Algorithm 2, would come early and hence increase the possibility of avoiding the constraint violation as compared to Algorithm 1 which, as noted in Figure 3, detects the constraint violation late. In general however, curve cutting is often actively chosen by the driver and thus becomes 
(a)

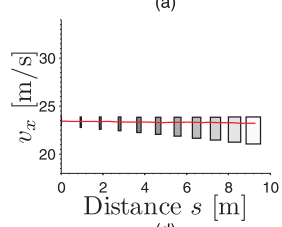

(d)
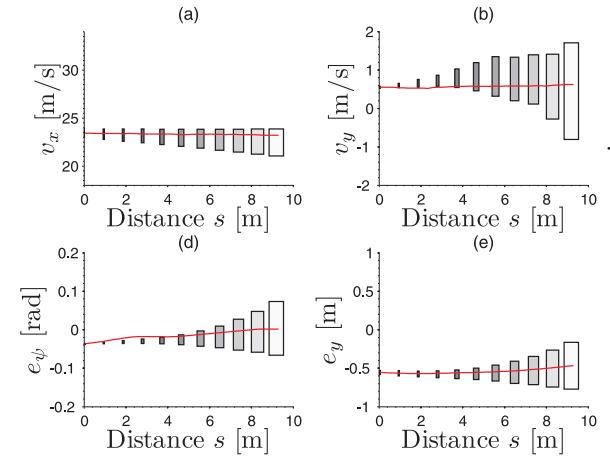

(c)

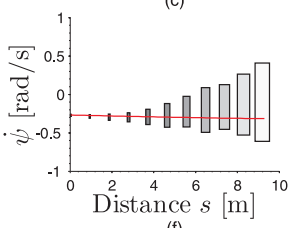

(f)

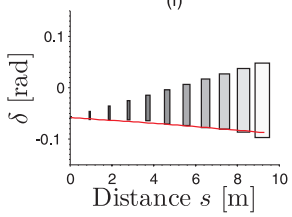

Fig. 7. (a)-(f) compare the approximated solution set $\hat{\Sigma}$ obtained through Algorithm 1 at position $b_{4}$ to measured vehicle states and steering angles. Each box shows predicted admissible variables for each step over the prediction horizon and the red solid line shows the actual trajectory traversed by the vehicle.

unavoidable very late. In such situations, the driver might thus perceive an early intervention as intrusive.

Let us instead consider the situation occurring between the time instances $c_{4}$ and $d_{5}$ where the relation between excessive speed and the constraint violations is more clear. At time $c_{4}$, the vehicle is traveling at a speed of approximately $85 \mathrm{~km} / \mathrm{h}$ and Algorithm 2 indicates that it is not possible to avoid a constraint violation within the prediction horizon by steering only. At time $b_{4}$, the vehicle speed has been slightly reduced to $84 \mathrm{~km} / \mathrm{h}$ and Algorithm 1 can still find a nonempty solution set $\hat{\Sigma}$. The obtained solution set $\hat{\Sigma}$ is reported in Figure 7 and indicates that constraint violations are potentially still avoidable through combined braking and steering. A safety intervention, triggered by Algorithm 2 might in this case have been considered unnecessary or at least early since no constraint violation occurred within the time interval $\left[c_{4}, c_{4}+N T_{s}\right]$. Nevertheless it turns out that the speed reduction adopted by the driver is insufficient. At time $a_{4}$, Algorithm 1 indicates that a constraint violation is no longer avoidable, after $<1 \mathrm{~ms}$ of computational time. At time $d_{4} \in\left[a_{4}, a_{4}+N T_{s}\right]$, the vehicle indeed violates the stability constraints (8), hence the constraint violation predicted by Algorithm 1 can be considered correct.

The experimental vehicle was equipped with an electronic stability control system which, in this situation, was activated. The stability control system can apply braking to individual wheels and is thus not restricted by the assumed control limitations of the driver. By braking individual wheels, additional yaw moment is generated by the stability system forcing the vehicle back in to the stable operating region. Keeping the vehicle in the lane is however not an objective of the electronic stability system. Instead, the driver needs to steer the vehicle correctly in order to stay in the lane. In this case, even though the vehicle is forced back in to the stable operating region, the stabilizing intervention combined with the driver's steering action does not keep the vehicle in the lane. At time $a_{5}$, Algorithm 1 recognizes that even though the vehicle is operating within the stable operating region, due to the position and motion of the vehicle, a violation of the constraints (7) is unavoidable. This took
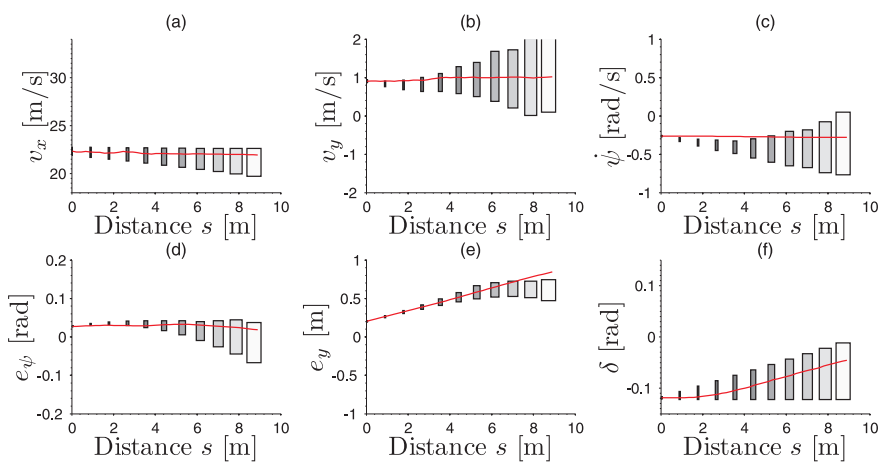

Fig. 8. (a)-(f) compare the approximated solution set $\hat{\Sigma}$ obtained through Algorithm 1 at position $b_{5}$ to measured vehicle states and steering angles. Each box shows predicted admissible variables for each step over the prediction horizon and the red solid line shows the actual trajectory traversed by the vehicle. (a)
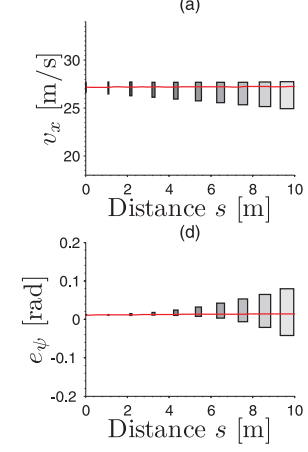

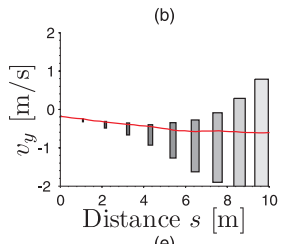

(e)

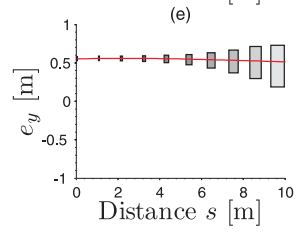

(c)
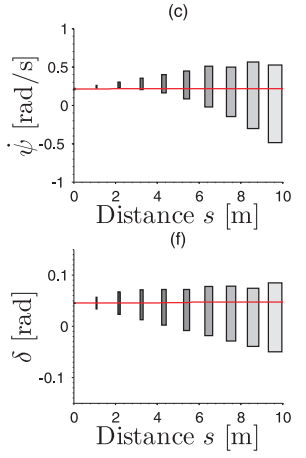

Fig. 9. (a)-(f) compare the approximated solution set $\hat{\Sigma}$ obtained through Algorithm 1 at position $b_{6}$ to measured vehicle states and steering angles. Each box shows predicted admissible variables for each step over the prediction horizon and the red solid line shows the actual trajectory traversed by the vehicle.

$28 \mathrm{~ms}$. At time $d_{5} \in\left[a_{5}, a_{5}+N T_{s}\right]$, the vehicle's front left corner indeed crosses the outer lane marking violating the constraints (7) as predicted by Algorithm 1. In this situation, both the violations of the stability constraints at time $d_{4}$ and the position constraints at time $d_{5}$, seems to be related to the excessive speed adopted when the vehicle approached the curve. In this case, a braking safety intervention issued based on either Algorithm 1 at the time instant $a_{4}$ or by Algorithm 2 at the time instant $c_{4}$ might have reduced the speed enough to avoid the constraint violations.

Finally we highlight that, in the considered dataset, no false constraint violations where indicated by Algorithm 1, while Algorithm 2, in some cases, predicted constraint violations which the driver managed to avoid by reducing speed. As an example, consider the point corresponding to the time instant $b_{6}$, shown in Figure 3 . In this point, the solution set obtained in Algorithm 2 is empty. Since no subsequent constraint violation occurs however, this can be considered a false threat detection. The solution set obtained with Algorithm 1 is on the other hand nonempty and is shown in Figure 9 . We note that the solution set obtained with Algorithm 1 encloses the actual trajectory traversed by the vehicle and that no intervention is needed in this scenario. 


\section{CONCLUDING REMARKS}

A model based threat assessment method, which accounts for combined braking and steering maneuvers in assessing the risk of unintended roadway departures has been presented and evaluated using experimental data. Compared to previously published approaches, which account for steering only, the present method reduces the risk of false threat detection while maintaining the ability to predict constraint violations. The preliminary results presented in this manuscript motivate further investigation of the algorithm's ability to predict constraint violations and the frequency of false threat detections.

\section{REFERENCES}

[1] J. Sandin and M. Ljung, "Understanding the causation of single-vehicle crashes: a methodology for in- depth on-scene multidisciplinary case studies," International Journal of Vehicle Safety (IJVS), vol. 2, no. 3, pp. 316333, 2007.

[2] M. Alirezaei, M. Corno, D. Katzourakis, A. Ghaffari, and R. Kazemi, "A Robust Steering Assistance System for Road Departure Avoidance," IEEE Transactions on Vehicular Technology, early access online, 2012.

[3] S. Glaser, S. Mammar, and C. Sentouh, "Integrated Driver Vehicle Infrastructure Road Departure Warning Unit," IEEE Transactions on Vehicular Technology, vol. 59, no. 6, pp. 2757-2771, 2010.

[4] P. Falcone, M. Ali, and J. Sjöberg, "Predictive Threat Assessment via Reachability Analysis and Set Invariance Theory," IEEE Transactions on Intelligent Transportation Systems, vol. 12, no. 4, pp. 1352-1361, 2011.

[5] G. S. Aoude, B. D. Luders, K. K. H. Lee, D. S. Levine, and J. P. How, "Threat assessment design for driver assistance system at intersections," In Proc. IEEE Intelligent Transportation Systems Conference, pp. 18551862, Sep. 2010.

[6] I. M. Mitchell, A. M. Bayen, and C. J. Tomlin, "A timedependent Hamilton-Jacobi formulation of reachable sets for continuous dynamic games," IEEE Transactions on Automatic Control, vol. 50, no. 7, pp. 947-957, Jul. 2005.

[7] I. M. Mitchell, "Application of level set methods to control and reachability problems in continous and hybrid systems," Ph.D., Stanford University, 2002.

[8] L. Jaulin, M. Kieffer, O. Didrit, and E. Walter, Applied interval analysis: with examples in parameter and state estimation, robust control and robotics. London: Springer, 2001.

[9] M. Bertozzi, A. Broggi, and A. Fascioli, "Vision-based intelligent vehicles: State of the art and perspectives," Robot. Auton. Syst., vol. 32, no. 1, pp. 1-16, Jul. 2000.

[10] H. B. Pacejka, Tyre and Vehicle Dynamics. Elsevier Science, 2005.

[11] S. Yamazaki, O. Furukawa, and T. Suzuki, "Study on Real Time Estimation of Tire to Road Friction," Vehicle System Dynamics, vol. 27, no. sup001, pp. 225-233, Jan. 1997.

[12] T. Shim and D. Margolis, "Model-Based Road Friction
Estimation," Vehicle System Dynamics, vol. 41, no. 4, pp. 249-276, Apr. 2004.

[13] F. Benhamou, F. Goualard, L. Granvilliers, and J. F. Puget, "Revising Hull and Box Consistency," Proceedings of the International Conference on Logic Programming, 1999.

[14] H. Collavizza, F. Delobel, and M. Rueher, "Comparing Partial Consistencies," Reliable Computing, vol. 5, no. 3, pp. 213-228, 1999.

[15] E. Gelso, M. Ali, and J. Sjöberg, "Threat Assessment for Driver Assistance Systems Using Interval-Based Techniques," in IFAC World Congress, Aug. 2011, pp. 97829787.

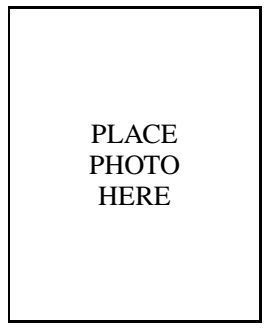

Mohammad Ali received the M.S. and Ph.D. degrees in 2005 and 2012, respectively, both from Chalmers University of Technology in Sweden.

Since 2005 he is with the Volvo Car Corporation, Sweden. He is currently conducting research and development at the Active Safety and Chassis department. His current research interests involve active safety, vehicle dynamics control and driving automation technologies.

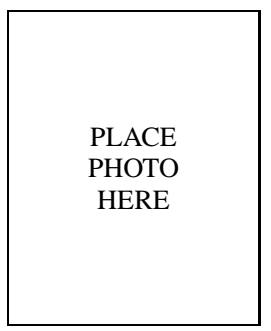

Esteban R. Gelso received the degree in electromechanical engineering from the National University of the Center of Buenos Aires Province, Argentina, in 2000, and the Ph.D. from the University of Girona, Spain, in 2009. During his Ph.D. studies he carried out research stays in Sweden, at Linköping University, in USA, at Vanderbilt University, and in Denmark, at Technical University of Denmark.

From 2009 to 2011, he was a Postdoctoral Fellow at Chalmers University of Technology, Sweden. Since 2011, he has been working as a Research Engineer at Volvo Group Trucks Technology - Advanced Technology and Research.

His research interests include the modeling, diagnosis, and control of complex dynamic systems, using interval-based and statistical-based techniques to handle uncertainty, with applications in different fields, and especially in the automotive industry.

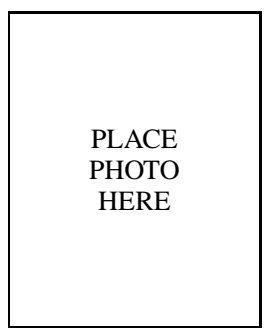

Jonas Sjöberg received the M.S. degree in engineering physics in 1989 from Uppsala University and the Ph. D. in 1995 from Linköping University, both in Sweden.

He has held visiting research positions at ETH Zurich, Switzerland, TU Wien, Austria, Technion, Haifa, Israel, and Vrije Universiteit, Brussels, Belgium. He has served as an Associate Editor for Control and Control Engineering Practice (1999-2008), and he regularly serves as international program committee member at international conferences. $\mathrm{He}$ is Professor at the Department of Signals and Systems, Chalmers, Gothenburg, Sweden, since 2001. He served as Programme Director of the Automation and Mechatronics education program between 2005 and 2010. His research interests are in Mechatronics, and Mechatronic related fields, such as Signal Processing, and Control. Within these fields, interest focus on model based methods, simulations, system identification, and optimization for design and product development of Mechatronic systems. Current applications are, for example, Automotive Active Safety and Hybrid Electric Vehicles. 